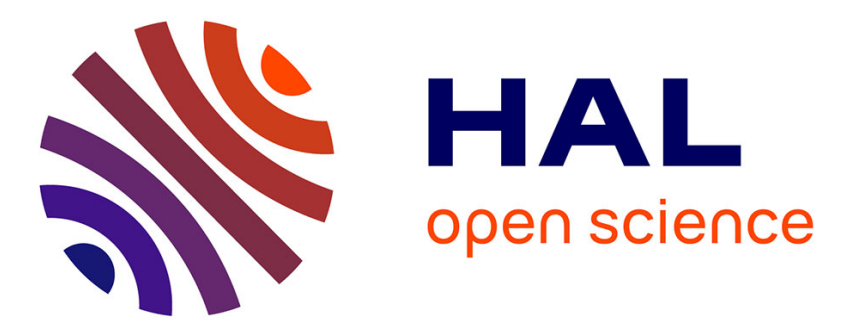

\title{
An experimental demonstration of coherent combining applied to optical parametric oscillators
}

\author{
Rodwane Chtouki, Pierre Bourdon, Anne Durécu, Laurent Lombard, \\ Christophe Planchat, Myriam Raybaut, Antoine Godard
}

\section{- To cite this version:}

Rodwane Chtouki, Pierre Bourdon, Anne Durécu, Laurent Lombard, Christophe Planchat, et al.. An experimental demonstration of coherent combining applied to optical parametric oscillators. SPIE LASE 2020, Feb 2020, San Francisco, United States. pp.112641B, 10.1117/12.2546022 . hal03185531

\section{HAL Id: hal-03185531 \\ https://hal.science/hal-03185531}

Submitted on 30 Mar 2021

HAL is a multi-disciplinary open access archive for the deposit and dissemination of scientific research documents, whether they are published or not. The documents may come from teaching and research institutions in France or abroad, or from public or private research centers.
L'archive ouverte pluridisciplinaire HAL, est destinée au dépôt et à la diffusion de documents scientifiques de niveau recherche, publiés ou non, émanant des établissements d'enseignement et de recherche français ou étrangers, des laboratoires publics ou privés. 


\title{
An experimental demonstration of coherent combining applied to optical parametric oscillators
}

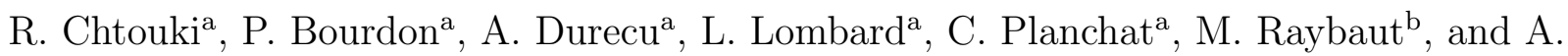 \\ Godard $^{\mathrm{b}}$

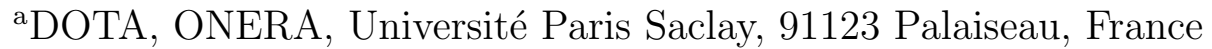 \\ ${ }^{b}$ DPHY, ONERA, Université Paris Saclay, 91123 Palaiseau, France
}

\begin{abstract}
Coherent beam combining (CBC) by active phase control could be useful for power scaling fiber-laser-pumped optical frequency converters like optical parametric oscillators (OPOs).

We developed an indirect phase control approach based on the phase matching relation intrinsic to efficient nonlinear processes. Previously, we demonstrated coherent combining of difference frequency generation through real time active control of the phases of the pump waves, using high bandwidth fibered electro-optic phase modulators. The straightforward follow-up is the application of such process to OPOs, higher efficiency frequency converters when compared to DFGs.

In this paper, we present an experimental demonstration of coherent OPOs emitting tunable idler wave in the mid-infrared. We present the architectures of continuous wave OPOs we are working on, their pros and cons and threshold properties, and the first results of coherent combining. We detail how the cavity modes of the OPOs are overlapped and how the active phase control used for DFG combining can be implemented in this case.
\end{abstract}

Keywords: Coherent Beam Combining, nonlinear optics, Optical Parametric Oscillators

\section{INTRODUCTION}

One way to overcome power limitations in fiber lasers induced by nonlinear effects such as stimulated Brillouin or Raman scattering, is to use coherent beam combining (CBC) techniques involving active phase control of the laser emitters. These have demonstrated their potential to power scale continuous-wave ${ }^{1}$ and pulsed $^{2}$ fiber lasers through coherent addition of the power emitted by multiple separate amplifiers, reaching overall emitted power exceeding the kilowatt level. ${ }^{1}$

The common wavelengths for high power fiber lasers are usually amongst $1 \mu m, 1.5 \mu m$ or $2 \mu m$. Higher ones can be obtained through nonlinear processes using nonlinear crystals in order to convert these laser lines. It is possible to obtain midinfrared waves presenting significant interest for gas monitoring, for instance around $4 \mu \mathrm{m}$. However the damage threshold of the nonlinear medium is a limiting factor for the power scaling of those frequency converters. With coherent combining techniques, it can be possible to overcome this limitation and to achieve higher output converted power. However, these methods require an active phase control, hence fast phase modulators at the midinfrared wavelength emitted by the converter. Such components are not exactly "off-theshelf" and are not as practical nor as fast as the all-fiber modulators available at standard laser wavelengths.

To solve this issue, it is possible to use the inherent phase-matching relation that lies in the nonlinear process, by an indirect control of the converted wave phase through control of the pump wave phase. When the pump comes from a fiber amplifier at the wavelength previously discussed, one can use off-the-shelf fibered phase modulators to obtain a frequency-converter coherent combining configuration. Our team successfully applied this approach to perform efficient coherent combination of second harmonic generators and difference frequency generators. ${ }^{3-5}$

Further author information:

R. Chtouki: E-mail: rodwane.chtouki@onera.fr, Telephone: +33 (0)1 80386334 
In this paper, we present the challenges of extending this indirect phase control technique to midinfrared OPO coherent combining. An experimental demonstration of OPO CBC is also detailed. Results will be presented during the conference.

\section{PRINCIPLE AND EXPERIMENTAL SETUP FOR CBC OF PARAMETRIC AMPLIFIERS}

\subsection{Coupled-Amplitude equation}

The demonstration of coherent beam combining of non-degenerate three waves mixing nonlinear processes was first made on difference frequency generators. The theoretical base is the same as for OPO CBC and it was a first step toward it, as both processes involve parametric converters.

As explained in the introduction, achieving $\mathrm{CBC}$ of frequency converters in the midinfrared requires phase control of one of the converted waves thanks to the phase-matching condition.

If we assume that the beams are linearly polarized, and use scalar expressions and equations, the electric field corresponding to each nonlinearly coupled wave can be written as:

$$
\widetilde{E}_{m}(z, t)=A_{m}(z) e^{j k_{m} z} e^{-j \omega_{m} t}+\text { c.c. }
$$

with $m=p, s$ or $i$ corresponding respectively to the pump, signal and idler wave. $A_{m}$ is the complex amplitude of the $\omega_{m}$ pulsation wave.

In the first millimeters of the nonlinear crystal, the parametric amplification is weak enough for us to assume that the pump wave is not depleted, which can then be extended through the crystal as the signal and idler waves generated during the firsts millimeters seed the rest of the crystal, leading to the description of the coupled-amplitude equation for frequency generation: ${ }^{6}$

$$
\left\{\begin{array}{l}
\frac{d A_{s}(z)}{d z}=\frac{8 \pi j \omega_{s}^{2} d_{e f f}}{k_{s} c^{2}} A_{p}(z) A_{i}^{*}(z) e^{j \Delta k z} \\
\frac{d A_{i}(z)}{d z}=\frac{8 \pi j \omega_{i}^{2} d_{e f f}}{k_{i} c^{2}} A_{p}(z) A_{s}^{*}(z) e^{j \Delta k z}
\end{array}\right.
$$

where $\Delta k=k_{p}-k_{s}-k_{i}$. The propagation of the idler wave can expressed as the following:

$$
\frac{d A_{i}(z)}{d z}=\kappa_{s}^{*} \kappa_{i}\left|A_{p}\right|^{2} A_{i}(z)
$$

with $\kappa_{m}=\frac{8 \pi j \omega_{m}^{2} d_{e f f}}{k_{m} c^{2}}$. Maximum power conversion is achieved for perfect phase-matching condition, i.e. $\Delta k=0$. This system of equations can be solved, in the case of an unseeded idler wave $A_{i}(0)=0$, assuming $\kappa=\sqrt{\kappa_{s}^{*} \kappa_{i}\left|A_{p}\right|^{2}}$ into:

$$
\left\{\begin{array}{l}
A_{s}(z)=A_{s}(0) \cosh (\kappa z) \\
A_{i}(z)=\frac{\kappa_{i} A_{3}}{\kappa} A_{s}^{*}(0) \sinh (\kappa z),
\end{array}\right.
$$

\subsection{Phase relation}

From these solutions, we see that the signal field keeps its initial phase-offset as it is only amplified by the process, whereas the idler wave phase-offset depends on both the pump wave's and the signal wave's:

$$
\varphi_{i}=\varphi_{p}-\varphi_{s}+\frac{\pi}{2}
$$

For quasi-phase matching (our case), where the nonlinear crystal is periodically poled, high conversion efficiency can be achieved. By propagating the wave in the crystal step by step in each domain, we can find the phase relation between the three waves to be:

$$
\varphi_{i}=\varphi_{p}-\varphi_{s}
$$


Thanks to this phase relation, it is possible to ensure coherent combining of the idler waves by sole control of the pump phase. If we consider two nonlinear processes in two different crystals, one called the reference, we have two phase relation as $\varphi_{i}^{r e f}=\varphi_{p}^{r e f}-\varphi_{s}^{r e f}$ and $\varphi_{i}^{n}=\varphi_{p}^{n}-\varphi_{s}^{n}$, having the two equal idler phases $\varphi_{i}^{r e f}=\varphi_{i}^{n}$ can also be expressed as:

$$
\varphi_{p}^{n}=\varphi_{p}^{r e f}-\varphi_{s}^{r e f}+\varphi_{s}^{n}
$$

If the pump phase $\varphi_{p}^{n}$ can be controlled to verify equation (9) at any time, the two idler phases will then be the same, hence the feasibility of $\mathrm{CBC}$ of frequency converters by indirect phase control. It is then possible to use fast and compact all-fiber electro-optics phase modulators (EOM).

\subsection{Experimental setup}

The experimental setup has been discussed in our previous paper. ${ }^{7}$ In order to demonstrate the experimental feasibility of such an indirect phase control, we have combined two DFG modules pumped by one $1.064 \mu \mathrm{m}$ Yb:fiber amplified lasers separated in two channels delivering up to $15 \mathrm{~W}$ each.

To obtain the DFG nonlinear process and to ensure the phase and wavelength stability of the signal, we used a 1553-nm laser diode amplified by a commercial erbium-doped fiber amplifier to seed both channels. This allows the idler wavelengths from the two channels to be equal, which is paramount for coherent combining. The experimental setup is presented in figure 1.

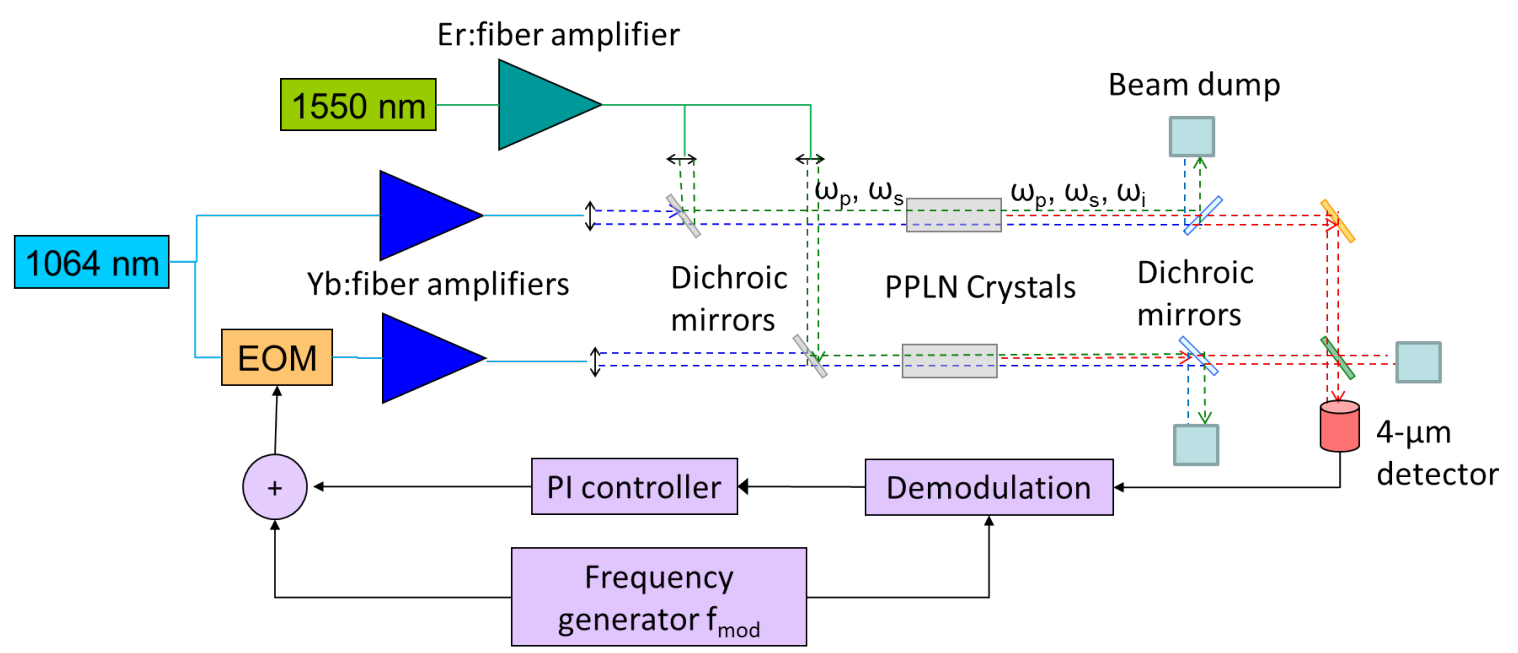

Figure 1. Schematics of the experimental setup for demonstrating CBC of two $3.4-\mu m$ difference frequency generators by phase-control of the $1.064 \mu \mathrm{m}$ pump wave. ${ }^{7}$

The DFG nonlinear crystals are PPLN crystal from Covesion and their length is $\mathrm{L}=20 \mathrm{~mm}$. With a 1064-nm pump wavelength and a seeded signal at $1553 \mathrm{~nm}$, quasi-phase matching in the PPLN crystal is obtained with a poling period of $30.49 \mu \mathrm{m}$ and at an operating temperature of $58^{\circ} \mathrm{C}$ for both crystals, generating an idler wave at $3.4 \mu \mathrm{m}$. The pump beams are focused down to $100 \mu \mathrm{m}$ waist in the PPLN crystals to maximize the conversion efficiency.

The phase error signal generation is obtained by using a commercial lock-in amplifier, thanks to a frequency tagging operating at $56 \mathrm{kHz}$. The EOM command the pump wave phase shift by a proportional integral (PI) controller allowing the phase error signal to be driven to 0 . 


\section{EXPERIMENTAL RESULTS AND LIMITS OF CBC OF DFG}

Coherent combining of these DFG modules is achieved using the idler waves interference signal to close the feedback loop (see figure 2). Time-averaged combining efficiency is excellent: the residual phase error is $\lambda / 28$ rms for the idler wave. This experiment is the proof that CBC of continuous wave DFG using indirect phase control is feasible.

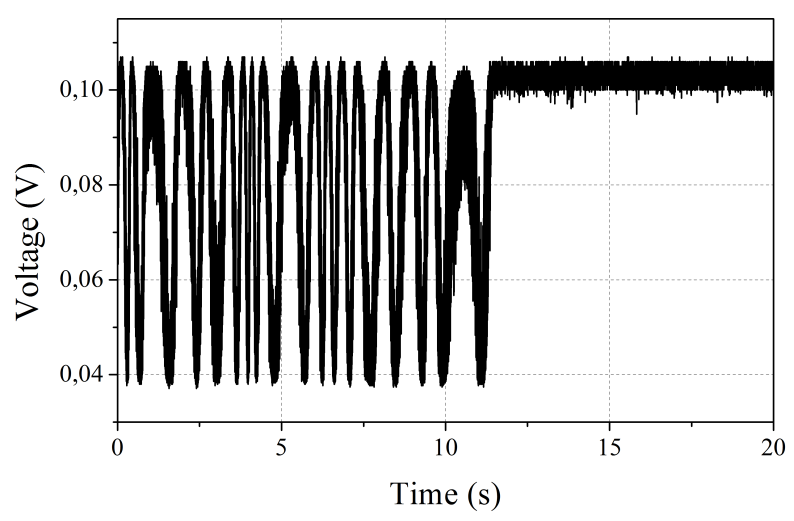

Figure 2. Interference signal of the DFG idler waves, fluctuating between destructive and constructive interference when $\mathrm{CBC}$ is inactive and stabilizing on a constructive interference state when phase-locking is active. ${ }^{7}$

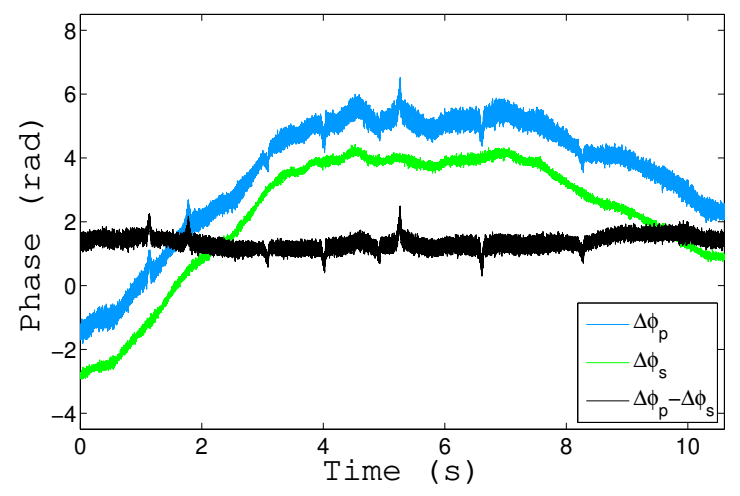

Figure 3. Measurement of the phase difference between the pump and signal waves when the phase control loop on the idler waves is closed. ${ }^{7}$

However, due to the low conversion efficiency of the DFG process (given in equation $(10)^{8}$ ), we could only use highly sensitive but slow midinfrared detector. This prevented the use of fast electronic in the demodulation setup and resulted in lower signal-to-noise ratio and higher residual phase error, as can be seen on figure 3. This low conversion efficiency also represents a limit in the practical use of CBC of DFG for potential devices as very low power of only a few $\mathrm{mW}$ is obtained.

$$
\eta=\frac{4 \omega_{i}^{2} d_{e f f} k_{p} k_{s}}{\pi c^{3} \varepsilon_{0} n_{p} n_{i} n_{s}\left(k_{p}+k_{s}\right)} h_{m}(B, \xi)
$$

Breaking records in terms of power was not the goal of the DFG CBC experiment previously presented. To obtain more power in the midinfrared, one can use longer crystals but will eventually be limited by the conversion efficiency or the damage threshold of the crystals. Another way is to use OPOs as they offer significantly higher conversion efficiency than a DFG module.

\section{TOWARD COHERENT COMBINING OF OPO}

\subsection{Interest of $\mathrm{CBC}$ of $\mathrm{OPO}$}

Optical parametric oscillators are a solution to overcome the issue of conversion efficiency as they take advantage of a cavity to increase the output idler power. They can reach tens of percents conversion efficiency for the idler wave, leading the way to potential device development with watts of power in the midinfrared.

Of the different existing configurations for an OPO, we chose to focus first on the singly resonant one, where only the signal wave resonates in the cavity. Continuous wave (CW) singly resonant OPOs (SROPO) can be useful for gas, molecular or ions spectroscopy. ${ }^{9}$ This choice was motivated by the fact that a SROPO offers both a high idler output power in the range of a few watts and a single cavity quite simple to stabilize and to control.

However this implies a higher oscillation threshold than for doubly resonant OPOs (DROPO), which is usually of only a few hundreds of milliwatts. Here we aimed for a configuration where the current experimental setup described in figure 1 can be easily modified to obtain the setup of figure 5 , i.e. with a pump power threshold around $10 \mathrm{~W}$. 


\subsection{Cavity design and threshold}

The pump power threshold for the SROPO can be expressed as the following: ${ }^{10}$

$$
P_{p, t h}=\frac{\varepsilon_{0} n_{s}^{2} c \lambda_{p}^{3}}{\pi^{2} d_{e f f}^{2} L\left(1-\delta^{2}\right) h_{m}(B, \xi)}\left(1-R_{s} e^{-\alpha_{s} L}\right),
$$

where $d_{\text {eff }}$ is the nonlinear coefficient of the crystal, $L$ its length, $h_{m}(B, \xi)$ the Boyd-Kleinman reduction factor with $\xi=L \lambda / 2 \pi n \omega_{0}^{2}, R_{s}$ the reflectivity of the cavity mirrors at the signal wavelength, $\alpha_{s}$ the absorption of the crytal and $\delta=2 \lambda_{p} / \lambda_{s}-1$.

Thanks to equation 11 it possible to evaluate the $1 / e^{2}$ beam radius that correspond to our experimental conditions. In order to obtain figure 4, we considered a ring-cavity OPO made of a $20 \mathrm{~mm}$ long PPLN crystal, with 2 plane dielectrics mirrors and 2 curved dielectrics mirrors with a $100 \mathrm{~mm}$ curvature radius. The nonlinear coefficient for PPNL crystal is $d_{e f f}=14 \mathrm{pm} / \mathrm{V}$, the reflectivity of the cavity mirrors is $R_{s}=98.8 \%$.

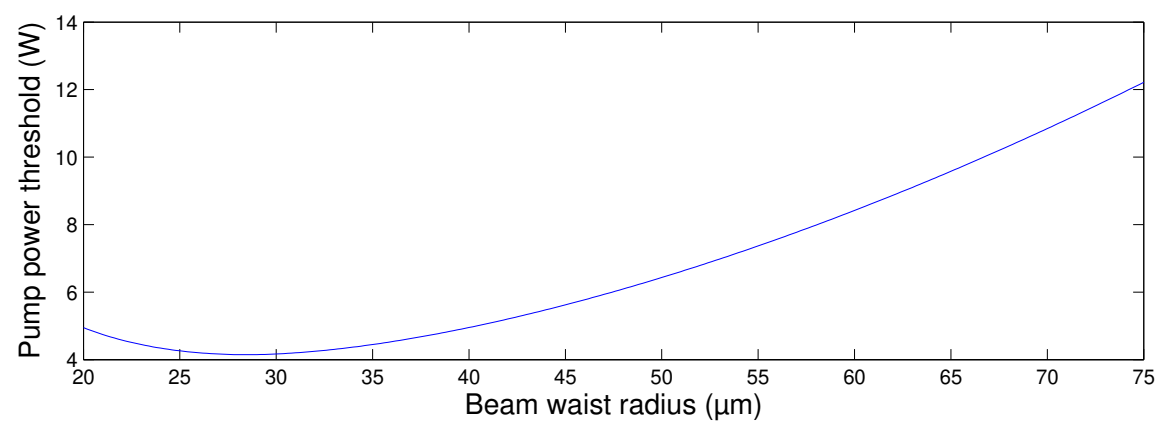

Figure 4. Evolution of the pump power threshold with the $1 / e^{2}$ beam radius.

\subsection{Experimental setup}

As explained previously, we aimed to use the same experimental setup as for CBC of DFG. A few changes appear as there is no signal seeding here, since the signal waves are amplified freely in the OPO cavities. Another change to mention is the use of a faster midinfrared detector as we have more idler power available, and thus the use of faster electronics for the demodulation and feedback-loop setup. This should result in a lower residual phase error and excellent combining efficiency. Where frequency tagging was limited to $56 \mathrm{kHz}$ in the previous experiment due to the detectors, we can now take advantage of faster detectors enabling $M H z$ frequency tagging.

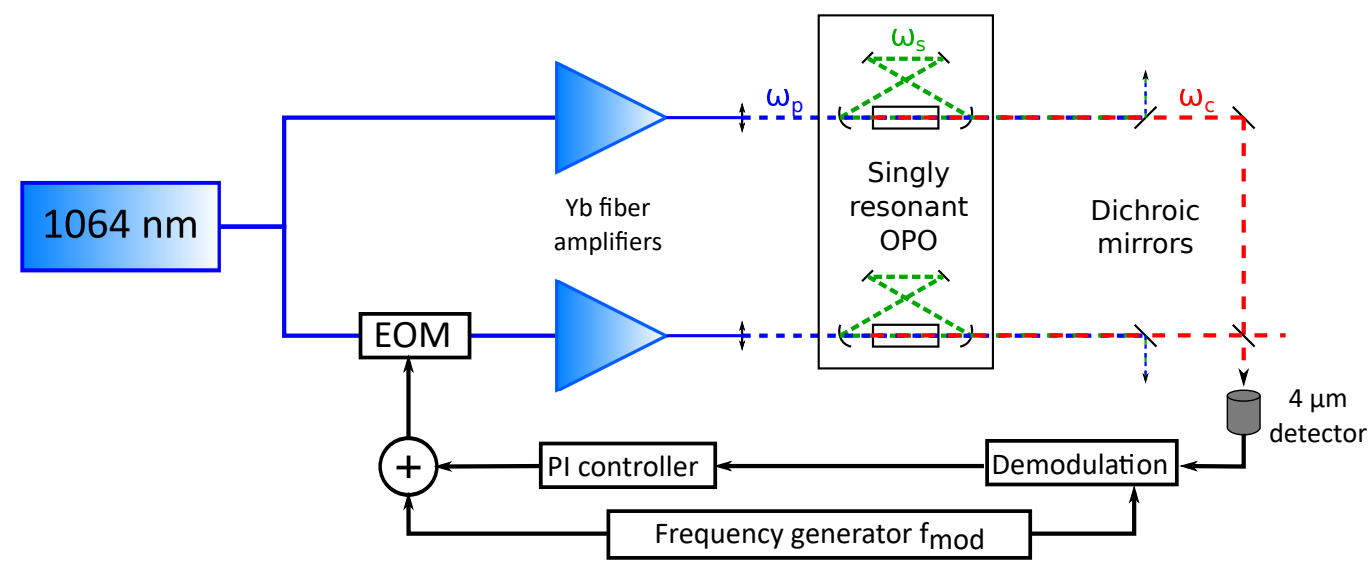

Figure 5. Schematics of the basic architecture required to combine two OPOs. 
The challenge of this setup lies within the balancing between two feedback loops. The first one is the one ensuring the indirect phase matching between the two idler waves, as done previously for DFG CBC. Since there is no seeding signal wave, the converted wavelengths are not always rigorously equal. This required stability for CBC can be achieved by tuning the length of the cavity with piezoelectric transducers (PZTs). Like for the first feedback loop, one of the two OPO idler wavelengths can fluctuate freely as the second one follows its changes thanks to the PZTs cavity length control. This represents a challenge as the two loops have to operate simultaneously without interfering with one another.

\section{CONCLUSION AND FUTURE WORK}

In this paper, the challenges of applying coherent combining by active phase control to much more powerful frequency converters, optical parametric oscillators (OPO), is presented. Optimizing the OPO threshold and coupling two different feedbacks are the main challenges in designing an optimal experiment. We also described the experimental demonstration of $\mathrm{OPO} \mathrm{CBC}$ we are now testing. The first results of this experiment will be presented during the conference.

\section{ACKNOWLEDGMENTS}

This work was partially funded by Delegation Generale pour l'Armement.

\section{REFERENCES}

[1] Flores, A., Shay, T. M., Lu, C. A., Robin, C., Pulford, B., Sanchez, A. D., Hult, D. W., and Rowland, K. B., "Coherent beam combining of fiber amplifiers in a kw regime," in [CLEO:2011 - Laser Applications to Photonic Applications], CLEO:2011 - Laser Applications to Photonic Applications, CFE3, Optical Society of America (2011).

[2] Lombard, L., Azarian, A., Cadoret, K., Bourdon, P., Goular, D., Canat, G., Jolivet, V., Jaouën, Y., and Vasseur, O., "Coherent beam combination of narrow-linewidth $1.5 \mu \mathrm{m}$ fiber amplifiers in a long-pulse regime," Opt. Lett. 36, 523-525 (Feb 2011).

[3] Odier, A., Durécu, A., Melkonian, J.-M., Lombard, L., Lefebvre, M., and Bourdon, P., "Coherent combining of second-harmonic generators by active phase control of the fundamental waves," Opt. Lett. 42, 3201-3204 (Aug 2017).

[4] Odier, A., Durécu, A., Melkonian, J.-M., Lombard, L., Lefebvre, M., and Bourdon, P., "Coherent combining of fiber-laser-pumped $3.4 \mu \mathrm{m}$ frequency converters," in [Fiber Lasers XIV: Technology and Systems], Robin, C. A. and Hartl, I., eds., 10083, 230 - 235, International Society for Optics and Photonics, SPIE (2017).

[5] Odier, A., Chtouki, R., Bourdon, P., Melkonian, J.-M., Lombard, L., Lefebvre, M., and Durécu, A., "Coherent combining of mid-infrared difference frequency generators," Opt. Lett. 44, 566-569 (Feb 2019).

[6] Boyd, R. W., [Nonlinear Optics, Third Edition], Academic Press, Inc., USA, 3rd ed. (2008).

[7] Bourdon, P., Chtouki, R., Durécu, A., Lombard, L., Planchat, C., Raybaut, M., and Godard, A., "Coherent combining of difference frequency generators: experimental demonstration and application to optical parametric oscillators," in [Nonlinear Frequency Generation and Conversion: Materials and Devices XVIII], Schunemann, P. G. and Schepler, K. L., eds., 10902, 70 - 76, International Society for Optics and Photonics, SPIE (2019).

[8] Zondy, J.-J., "The effects of focusing in type-i and type-ii difference-frequency generations," Optics Communications 149(1), 181 - 206 (1998).

[9] Courtois, J., Bouchendira, R., Cadoret, M., Ricciardi, I., Mosca, S., Rosa, M. D., Natale, P. D., and Zondy, J.-J., "High-speed, multi-thz-range mode-hop-free tunable mid-ir opo spectrometer," in [Advanced Solid-State Lasers Congress], Advanced Solid-State Lasers Congress, JTh2A.19, Optical Society of America (2013).

[10] Sutherland, R., [Handbook of Nonlinear Optics], Optical Science and Engineering, Taylor \& Francis (2003). 